\title{
BMJ Open Mortality and use of psychotropic medication in patients with stroke: a population-wide, register-based study
}

\author{
Poul Jennum, ${ }^{1}$ Lone Baandrup, ${ }^{2}$ Helle K Iversen, ${ }^{3}$ Rikke Ibsen, ${ }^{4}$ Jakob Kjellberg ${ }^{5}$
}

To cite: Jennum $P$, Baandrup L, Iversen HK, et al. Mortality and use of psychotropic medication in patients with stroke: a population-wide, registerbased study. BMJ Open 2016;6:e010662. doi:10.1136/bmjopen-2015010662

- Prepublication history for this paper is available online. To view these files please visit the journal online (http://dx.doi.org/10.1136/ bmjopen-2015-010662).

Received 25 November 2015 Revised 26 January 2016 Accepted 9 February 2016

CrossMark

For numbered affiliations see end of article.

Correspondence to Dr Poul Jennum; poul. joergen.jennum@regionh.dk

\section{ABSTRACT}

Objectives: The study sought to describe whether psychotropic medication may have long-term side effects in patients with stroke compared with controls.

Setting: Use of national register data from healthcare services were identified from the Danish National Patient Registry in Denmark. Information about psychotropic medication use was obtained from the Danish Register of Medicinal Product Statistics.

Objectives: We aimed to evaluate all-cause mortality in relation to the use of benzodiazepines, antidepressants and antipsychotics in patients with stroke and matched controls.

Participants: Patients with a diagnosis of stroke and either no drug use or preindex use of psychotropic medication $(n=49968)$ and compared with control subjects ( $n=86100)$ matched on age, gender, marital status and community location.

Primary outcome measure: All-cause mortality. Results: All-cause mortality was higher in patients with previous stroke compared with control subjects. Mortality HRs were increased for participants prescribed serotonergic antidepressant drugs ( $H R=1.699$ ( $S D=0.030), p=0.001$ in patients; $H R=1.908$ (0.022), $p<0.001$ in controls, respectively), tricyclic antidepressants ( $H R=1.365$ (0.045), $p<0.001$; $\mathrm{HR}=1.733(0.022), p<0.001)$, benzodiazepines $(H R=1.643(0.040), p<0.001 ; H R=1.776(0.053)$, $p<0.001)$, benzodiazepine-like drugs ( $H R=1.776$ (0.021), $p<0.001 ; H R=1.547$ (0.025), $p<0.001)$, firstgeneration antipsychotics ( $\mathrm{HR}=2.001(0.076), \mathrm{p}<0.001$; $\mathrm{HR}=3.361$ (0.159), $p<0.001$ ) and second-generation antipsychotics (HR=1.645 (0.070), $p<0.001 ; H R=2.555$ $(0.086), p<0.001)$, compared with no drug use. Interaction analysis suggested statistically significantly higher mortality HRs for most classes of psychotropic drugs in controls compared with patients with stroke.

Conclusions: All-cause mortality was higher in patients with stroke and controls treated with benzodiazepines, antidepressants and antipsychotics than in their untreated counterparts. Our findings suggest that care should be taken in the use and prescription of such drugs, and that they should be used in conjunction with adequate clinical controls.

\section{INTRODUCTION}

Stroke is one of the most important neurological causes of morbidity, mortality and

\section{Strengths and limitations of this study}

- Hypnotics, antidepressants and antipsychotics are more frequently used in patients with stroke than in controls and are associated with greater all-cause mortality in patients with stroke and matched controls.

- Care should be taken in the use and prescription of such drugs, and they should be used in conjunction with adequate clinical control.

- The strengths of the National Patient Registry include the following: it is a national database that includes all identified patients and comorbid conditions and psychotropic medication, it is time locked (all reports must be associated with patient contacts) and includes a substantial follow-up period.

- The limitation of the study is that although the study is controlled, it is not a randomised controlled trial, which means there is a potential bias towards more severe diseases among treated patients.

disability and affects a significant proportion of the population. Stroke has deleterious effects on patients' social function, employment and quality of life, and the disease entails a societal burden that is manifested as increased direct and indirect costs. ${ }^{1}$ Early intervention and management of stroke in recent years have significantly improved prognosis, with reduced mortality and fewer neurological deficits. After the acute phase, up to $40 \%$ of patients suffer from post-stroke depression within the first year, ${ }^{2}$ and patients may suffer from comorbid insomnia and psychiatric symptoms, such as hallucinations and other psychotic symptoms. ${ }^{3}$

In recent years, the increased frequency of off-label use of psychotropic drugs, their side effects and the harm they may cause has been a growing concern. ${ }^{4-6}$ Studies of large groups of patients with psychotic diseases and of patients with chronic insomnia have raised concerns about increased mortality rates in benzodiazepine (BZD)-treated 
patients, especially when used in combination with other psychotropic drugs. ${ }^{7-9}$ There is additional concern about the association between BZD use and cognitive decline in Alzheimer's disease ${ }^{10-13}$ Patients with stroke suffer from major comorbidities, including cardiac arrhythmias and sleep-related hypoxaemia. ${ }^{14}$ Few studies have addressed the effect on mortality when using antidepressants in patients with post-stroke. These studies have found reduced mortality rates in short-term studies in patients treated during the stable phase ${ }^{15}$ and there is also some evidence that the use of selective serotonin reuptake inhibitors (SSRIs) may improve recovery after stroke. ${ }^{16}$ However, there have been no prospective studies of morbidity and mortality in patients with stroke in relation to the use of psychotropic drugs.

The aim of this study was to evaluate the association between all-cause mortality and the use of BZDs, BZD-like medications, tricyclic antidepressants (TCAs), SSRIs, serotonin-norepinephrine reuptake inhibitors (SNRIs) and first-generation and second-generation antipsychotics (FGAs and SGAs) in patients with stroke and matched controls.

\section{MATERIALS AND METHODS}

Subjects

In Denmark, all hospital contacts are recorded in the National Patient Registry (NPR) with respect to the time of contact and information about primary and secondary diagnoses. The NPR includes administrative information and details on all diagnoses, diagnostic methods, hospitalisations and ambulatory clinical contacts (initial and follow-up) in all public and private hospitals. These data are recorded using several international classification systems, including the International Classification of Diseases, 10th edition (ICD-10). The NPR is a timebased, nation-wide register that includes data from all inpatient and outpatient contacts. Thus, the data extracted for this study are representative of all patients in Denmark who have been diagnosed with stroke, irrespective of other diagnoses. Data were available for the entire observation period; thus, we were able to trace patients retrospectively and prospectively, relative to the time of their diagnosis.

Using the NPR, we identified all patients diagnosed with stroke between 1997 and 2009. To identify stroke diagnoses, we used the ICD-10 codes; I61: cerebral haemorrhage, I63: brain infarction and I64: stroke not otherwise defined as being due to infarction or haemorrhage. We observed 76591 cases in the total period. For those compliant 69970 cases and 49968 survived 1 year. The diagnosis code is recorded after patient evaluation in each hospital (based on a standardised evaluation of stroke). The expected distribution of stroke types is $15 \%$ haemorrhagic and $85 \%$ ischaemic. ${ }^{17}$ We did not include patients with a diagnosis of transitory ischaemic episodes. Then, using data from Denmark's Civil Registration System Statistics, we randomly selected citizens of the same age, gender, county of residence and marital status as the patients, but who did not have a diagnosis of any stroke episodes. Parity of socioeconomic status (SES) was ensured by selecting control subjects from the same part of the country in which the patient lived and by matching for partnership (including those married). A ratio of control subjects to patients of 2:1 was used to take into account the variation among the controls. Data from patients and matched controls that could not be identified in the Coherent Social Statistics database were excluded from the sample. The patients and matched control subjects were traced retrospectively and prospectively for up to 12 years in the NPR registry and prescription database. All observations were successfully matched at the year of diagnosis (index year). Patients and control subjects who could not be identified in the social security registry in the years before and after diagnosis (typically as a result of emigration) were excluded from the sample. More than $99 \%$ of the patients and controls had complete data.

\section{Psychotropic medications studied}

Information on type, number, dose and date of prescriptions was obtained from the Danish Register of Medicinal Product Statistics, which includes all medications prescribed outside hospitals. Seven medication classes were included in the analysis: BZDs, BZD-like drugs, SSRIs, SNRIs, TCAs, FGAs and SGAs. Medication use was defined as filling of at least three prescriptions with a compliance rate of at least $60 \%$ in the 12 months before the index date. Compliance rate was defined as the daily defined dose (DDD) coverage of prescriptions and was set to $60 \%$ as an inclusion criterion. The no-medication group was defined as those who had not collected any psychotropic drugs within 12 months prior to the index date or during the observation period. The compliance was determined in the preperiod for the compliant group. Non-compliant was defined as not meeting the compliance goal but collecting medication in the preperiod, and they were excluded from the population. Non-user did not collect any medication in either the preperiod or postperiod, so they are true non-users.

A substantial number of patients used medication before the year of diagnosis. We created a dummy variable to control for this in the regression. Some patients discontinued medication; they were identified by determining whether the most recent prescription had been collected more than 3 years before the end point (stop parameter). Such cases were scored as 0 in the analysis.

\section{Statistical analyses}

Morbidity and mortality data were extracted as primary and secondary diagnoses from the NPR in accordance with the WHO ICD-10 criteria. For the purpose of this study, we used all-cause morbidity and all-cause mortality data. To avoid the influence of mortality due to factors 
other than medication use, we only included patients and controls who had survived for at least 1 year after the index date and who were treated with not more than one psychotropic medication to avoid the influence of the others. Statistical analysis involved the development of proportional Cox hazard models in which survival was conditional on the explanatory variables of age, gender and use of medication types. Three regressions were performed for the case and control groups separately, and for both groups. In the latter, a dummy variable indicating case versus control and interaction terms for medication $\times$ control were included. The interaction term should indicate whether the effect of that particular medication on survival was different for the case and control groups. After conditioning on 1 year of survival (removing both cases and controls from the population), we tested the model for proportionality to make sure that the assumption for the proportional Cox model was met. Since this was not the case for some covariates, an extended Cox model was developed, using a timexinteraction term for the covariates that were not proportional. The interpretations of the covariates were not straightforward once the interaction with time had been included. The effect of the non-proportional covariates on survival could be calculated for each year after the index date. Statistical analyses were performed using SAS V.9 (SAS, Inc, Cary, North Carolina, USA).

\section{RESULTS}

In total, the study population comprised 49968 patients with ischaemic, haemorrhagic or unspecified stroke and 86100 controls. The descriptive information for the study population is shown in table 1 . Patients with stroke suffered from high preindex (ie, 12 months prior to the index date) mortality.

The distribution of the patients and control subjects along with their treatment is shown in table 1. In general, patients with stroke were more frequently treated with a psychotropic drug than were the controls (table 1).

\section{Survival after a stroke diagnosis}

Survival data with respect to total preindex medication use in patients with stroke and their controls are shown in figure 1 subdivided into those treated or not treated with any psychotropic drugs. Mortality HRs for each class of medication are shown in table 2 and the results of the Cox model with interactions in table 3 adjusted for the effect of age, gender and Charlson comorbidity index. Survival was generally lower, with increased mortality HRs, for all drug classes in patients with stroke and controls. We found statistically significant interactions between group and psychotropic medication regarding TCAs, SSRIs, BZD-like drugs and SGAs (table 3), that is, the associations of excess risk of death with use of these classes of psychotropic medications were more pronounced in control subjects compared with patients
Table 1 Number of the total population conditioned on 1 year of survival after the first onset of stroke, classified as patients with stroke and their controls, and their distribution with respect to psychotropic drug use

\begin{tabular}{|c|c|c|c|c|c|}
\hline & \multicolumn{2}{|c|}{$\begin{array}{l}\text { Patients with } \\
\text { stroke }\end{array}$} & \multicolumn{2}{|c|}{ Controls } & \multirow[b]{2}{*}{ p Value } \\
\hline & $\mathbf{N}$ & $\begin{array}{l}\text { Per } \\
\text { cent }\end{array}$ & $\mathbf{N}$ & $\begin{array}{l}\text { Per } \\
\text { cent }\end{array}$ & \\
\hline Age (mean, SD) & 66.6 & 14.4 & 65.3 & 14.5 & \\
\hline Gender (female) & 21995 & 44.0 & 36595 & 42.5 & \\
\hline No medication & 33778 & 67.6 & 72707 & 84.4 & $<0.001$ \\
\hline SSRI/SNRI & 8366 & 16.7 & 6789 & 7.9 & $<0.001$ \\
\hline TCA & 1082 & 2.2 & 756 & 0.9 & $<0.001$ \\
\hline BZD & 4675 & 9.4 & 3542 & 4.1 & $<0.001$ \\
\hline Z-drugs & 5165 & 10.3 & 4331 & 5.0 & $<0.001$ \\
\hline FGA & 381 & 0.8 & 349 & 0.4 & $<0.001$ \\
\hline SGA & 511 & 1.0 & 421 & 0.5 & $<0.001$ \\
\hline $\begin{array}{l}\text { Charlson } \\
\text { comorbidity } \\
\text { index } \\
\text { (mean, SD) }\end{array}$ & 0.8 & 0.8 & 0.1 & 0.4 & $<0.001$ \\
\hline All & 49968 & & 86100 & & \\
\hline
\end{tabular}

with stroke. The HRs were highest among controls using any of the psychotropic drugs compared with patients with stroke (table 3).

\section{DISCUSSION}

This study, which included data from a nation-wide patient registry, yielded several important findings: (1) psychotropic drugs are more frequently used by patients with stroke than by matched control individuals; (2) psychotropic drugs are generally associated with increased mortality, the highest mortality being observed in patients with stroke and control subjects treated with antipsychotics; (3) control subjects showed higher mortality HRs (SSRI, TCA and BZD-like drugs) compared with patients with stroke and (4) this association is present after adjusting for comorbidities and avoiding the initial phase with high mortality which may be due to the stroke and multiple comorbidities immediately after the stroke.

The higher mortality with the use of BZDs, antidepressants and antipsychotics in patients with stroke was not exclusively associated with the presence of stroke because the association was also found in control subjects. Previous studies have evaluated the mortality rates associated with BZDs in patients with other conditions, including patients with insomnia, ${ }^{78}$ patients in dialysis ${ }^{18}$ and those with depression. ${ }^{19}$ The need for further evaluation of the use of antidepressants and antipsychotics and their associations with morbidity, including metabolic syndrome and mortality in other groups of patients 


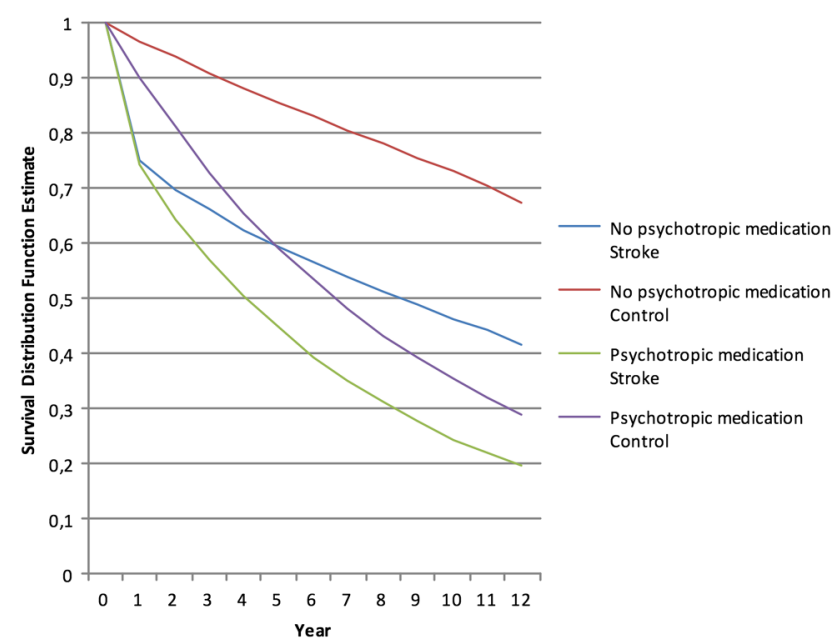

Figure 1 Survival by groups: total patients with stroke versus total controls, and patients with stroke versus controls subdivided into those treated or not treated with any psychotropic drugs, by years of follow-up.

including psychiatric, neurodegenerative and cardiac diseases, has been stressed by many authors. ${ }^{20-24} \mathrm{~A}$ major concern about studies of this type is whether the group of people receiving treatment is a population with intrinsically higher comorbidity rates than the untreated population. We cannot discount this possibility in the population considered here. Depression is associated with an increased risk of developing cardiac and cerebrovascular events, ${ }^{25}{ }^{26}$ and with new events in patients with established cardiovascular disease. ${ }^{27}$ Patients with stroke are more likely to be treated for depression ${ }^{28-30}$ and suffer from insomnia ${ }^{3}$ and depressive symptoms. ${ }^{31} 32$ Earlier studies addressed the potentially greater mortality in patients with stroke using antipsychotics. ${ }^{32-34}$ All-cause mortality is increased in psychiatric diseases, the highest rates are found among patients with psychosis, followed by depression and anxiety. ${ }^{35}$ The increase in mortality in this study may be related to the underlying psychiatric disorder rather than to its treatment. In a recent study, lower mortality was found among patients with schizophrenia with moderate antipsychotic and antidepressant medication but higher mortality when treated with BZDs. ${ }^{36}$ These findings suggest that drug-related mortality may depend on the psychiatric or neurological disorder in question. Although not proving that comorbid conditions play a role in the higher mortality of those treated with psychotropic drugs, it does suggest that the relation is associated with effect of the drugs. We included three major classes of psychotropic drugs in this study to evaluate the effects of their individual use. We also included the use of BZD-like drugs as it has previously been assumed that these drugs are safer than BZDs. We found no such effect; there was no major difference in mortality between BZD and BZD-like drugs. This population is one of the largest ever studied, but, even though we found that the risk was still higher for SSRIs, BZD-like drugs and antipsychotics, our results suggest that even larger population-based studies, with longer follow-up times, are required. This is reflected in table 3, which illustrates a subgroup analysis of specific groups of drugs that attempts to evaluate the different types. Further subclassification of morbidities that adequately control for different types of psychiatric and medical diagnosis would require an even larger dataset.

There are several factors that could explain why some of the drugs studied might affect morbidity and even mortality rates. The drugs may affect arousals from sleep, metabolic, respiratory, autonomic and cardiac function. ${ }^{37-39}$ BZDs may induce upper airway collapse and potentially affect respiration. Use of clonazepam has been reported to induce sleep apnoea. ${ }^{40}$ This is potentially important as sleep apnoea is very common in

Table 2 HRs of mortality in patients with stroke and control subjects

\begin{tabular}{|c|c|c|c|c|c|c|}
\hline & \multicolumn{3}{|c|}{ Patients with stroke } & \multicolumn{3}{|c|}{ Controls } \\
\hline & HR & SD & p Value & HR & SD & p Value \\
\hline Age & $1.087^{\star}$ & $<0.001$ & $<0.001$ & 1.114 & $<0.001$ & $<0.001$ \\
\hline Gender (female) & 0.786 & 0.016 & $<0.001$ & 0.679 & 0.017 & $<0.001$ \\
\hline Charlson comorbidity index & $1.076^{\star}$ & 0.014 & $<0.001$ & $1.403^{\star}$ & 0.014 & $<0.001$ \\
\hline SSRI (incl. SNRI) & $1.699^{\star}$ & 0.030 & $<0.001$ & 1.908 & 0.022 & $<0.001$ \\
\hline TCA & 1.365 & 0.045 & $<0.001$ & 1.733 & 0.060 & $<0.001$ \\
\hline BZD & $1.643^{\star}$ & 0.040 & $<0.001$ & $1.776^{\star}$ & 0.053 & $<0.001$ \\
\hline BZD-like drugs & 1.386 & 0.021 & $<0.001$ & 1.547 & 0.025 & $<0.001$ \\
\hline FGA & 2.001 & 0.076 & $<0.001$ & $3.361^{*}$ & 0.159 & $<0.001$ \\
\hline SGA & 1.645 & 0.070 & $<0.001$ & 2.555 & 0.086 & $<0.001$ \\
\hline Observations & 49968 & & & 86100 & & \\
\hline Per cent censored & 64.5 & & & 81.3 & & \\
\hline \multicolumn{7}{|c|}{$\begin{array}{l}\text { Bold typeface indicates } p<0.001 \text {. } \\
\text { Timexinteraction coefficient not reported. } \\
\text { Estimate=a+a_txyear. } \\
\text { *Time-varying covariates were calculated in year 2, when all time-dependent covariates were significant in the time test. } \\
\text { BZD, benzodiazepine; FGA, first-generation antipsychotic; SGA, second-generation antipsychotic; SNRI, serotonin-norepinephrine reuptake } \\
\text { inhibitor; SSRI, selective serotonin reuptake inhibitor; TCA, tricyclic antidepressant. }\end{array}$} \\
\hline
\end{tabular}


Table 3 Mortality HRs for psychotropic drugs in the total study sample

\begin{tabular}{|c|c|c|c|c|c|c|}
\hline & \multicolumn{3}{|c|}{ Basic Cox model $^{*}$} & \multicolumn{3}{|c|}{ Cox model with interactions* } \\
\hline & HR & SD & p Value & HR & SD & p Value \\
\hline Age & 1.102 & 0.001 & $<0.001$ & 1.102 & 0.001 & $<0.001$ \\
\hline Gender (female) & 0.734 & 0.012 & $<0.001$ & 0.734 & 0.012 & $<0.001$ \\
\hline Control group & 0.600 & 0.013 & $<0.001$ & 0.547 & 0.015 & $<0.001$ \\
\hline Charlson comorbidity index & 1.175 & 0.010 & $<0.001$ & 1.169 & 0.010 & $<0.001$ \\
\hline SSRI (incl. SNRI) & 1.737 & 0.014 & $<0.001$ & 1.270 & 0.029 & $<0.001$ \\
\hline TCA & 1.500 & 0.036 & $<0.001$ & 1.404 & 0.045 & $<0.001$ \\
\hline BZD & 1.720 & 0.032 & $<0.001$ & 1.532 & 0.023 & $<0.001$ \\
\hline BZD-like drugs & 1.536 & 0.027 & $<0.001$ & 1.340 & 0.021 & $<0.001$ \\
\hline FGA & 2.374 & 0.057 & $<0.001$ & 2.260 & 0.076 & $<0.001$ \\
\hline SGA & 1.934 & 0.054 & $<0.001$ & 1.745 & 0.070 & $<0.001$ \\
\hline \multicolumn{7}{|l|}{ Interactions } \\
\hline ControlxSSRI & & & & 1.165 & 0.045 & $<0.001$ \\
\hline ControlxTCA & & & & 1.209 & 0.075 & 0.011 \\
\hline Control×BZD & & & & 1.164 & 0.057 & 0.143 \\
\hline Control×BZD-like drugs & & & & 1.236 & 0.032 & $<0.001$ \\
\hline Control×FGA & & & & 1.125 & 0.115 & 0.305 \\
\hline Control×SGA & & & & 1.327 & 0.111 & 0.011 \\
\hline Observations & 136068 & & & 136068 & & \\
\hline Per cent censored & 75.1 & & & 75.1 & & \\
\hline
\end{tabular}

Bold typeface indicates $p<0.001$.

Time-varying covariates were calculated in year 2, when all time-dependent covariates were significant in the time test.

Estimate $=a+a \_t \times y e a r$.

*Time interaction coefficient not reported.

BZD, benzodiazepine; FGA, first-generation antipsychotic; SGA, second-generation antipsychotic; SNRI, serotonin-norepinephrine reuptake inhibitor; SSRI, selective serotonin reuptake inhibitor; TCA, tricyclic antidepressant.

patients with stroke. ${ }^{3}$ The respiratory depression of hypnotics has not been fully identified in polysomnography studies. ${ }^{41}$ These studies are performed under controlled circumstances in a small number of selected patients, while natural usage includes unselected patients in their natural environment, where dose and presence of other risk factors (eg, comedication, sleep deprivation and alcohol consumption) are not controlled for. Overdoses and combinations have been reported in patients admitted to emergency rooms. ${ }^{42}$ Furthermore, predictors of higher mortality include male gender, increasing age and lower educational level, combined with alcohol consumption and inappropriate lifestyles-factors that may be associated with the use of psychotropic drugs and that are not taken into consideration in clinical laboratory testing. Another Danish study confirmed our findings of higher total mortality but lower cardiovascular mortality among SSRI-treated patients with stroke. It was concluded that this was due to the higher haemorrhagic mortality in treated patients. ${ }^{43}$ Consequently, future studies should explore the relationship between psychotropic drugs, morbidity and mortality in greater detail.

In this study, we carried out a total evaluation, controlling for gender, age and demographic variables, which are all known to influence disease outcome, and for comorbidities. We also avoided the initial period when patients are much frailer and mortality rates are high due to the direct effect of the stroke, and the great comorbidity in these patients. As such, we believe that the risk associations found in the study are conservative, as is further supported by the finding that the risk association in patients with stroke is lower than in controls.

The study has a number of limitations: (1) it is based on clinic/hospital reports to the NPR; (2) the diagnostic accuracy depends on the clinic's presentation and reporting of the diagnosis and any comorbidities; (3) confounder variables (eg, BMI and other cardiovascular risk factors) were not recorded; and (4) symptoms and clinical evaluation results were not considered (eg, those measured by polysomnography, limited channel polygraphy and cardiac evaluations), so we could not relate our findings to disease severity. We did not exclude diseases, especially unidentified stroke, in the controls. The types of medication given on prescription are highly controlled in Denmark, but we do not know whether the drugs were taken or shared, or if they were taken from someone else's prescription (eg, that of the patient's spouse). Nevertheless, we believe that using at least three prescriptions indicates more chronic use. We have no definitive explanation for the higher mortality rate among the controls compared with the patients with stroke: one cause could be that we only included patients who survived after 1 year. Evaluation of the total population showed smaller differences, so it is likely that those who survived the stroke incident have higher survival rates despite the treatment with psychotropic drugs.

The strengths of the NPR, however, are that it is a national database that includes all identified patients and 
comorbid conditions and psychotropic medication, it is time locked (all reports must be associated with patient contacts) and includes a substantial follow-up period. In addition, although the study is controlled, it is not a randomised controlled trial, which means there is a potential bias towards more severe diseases among treated patients.

We conclude that use of hypnotics, antidepressants and antipsychotics is more frequently used preindex in patients with stroke than in controls, and is associated with greater all-cause mortality in patients with stroke and matched controls.

\section{Author affiliations}

${ }^{1}$ Faculty of Health Sciences, Danish Center for Sleep Medicine, Neurophysiology Clinic, University of Copenhagen, Rigshospitalet, Copenhagen, Denmark

${ }^{2}$ Center for Neuropsychiatric Schizophrenia Research \& Center for Clinical Intervention and Neuropsychiatric Schizophrenia Research, Mental Health Center Glostrup, Copenhagen University Hospital, Glostrup, Denmark ${ }^{3}$ Stroke Unit, Department of Neurology, Faculty of Health Sciences, University of Copenhagen, Rigshospitalet, Copenhagen, Denmark

${ }^{4}$ i2minds, Aarhus, Denmark

${ }^{5}$ Danish National Institute for Local and Regional Government Research, Copenhagen, Denmark

Acknowledgements Centre for Healthy Aging, Faculty of Health Sciences, University of Copenhagen.

Contributors PJ and JK created, initiated and managed the project. JK and R performed the statistical analyses and commented on the manuscript. LB and $\mathrm{HKI}$ commented on the methods and critically revised the manuscript.

Funding This research received no specific grant from any funding agency in the public, commercial or not-for-profit sectors.

Competing interests None declared.

Ethics approval Register Study, Danish Data Protection Agency acceptance.

Provenance and peer review Not commissioned; externally peer reviewed.

Data sharing statement No additional data are available.

Open Access This is an Open Access article distributed in accordance with the Creative Commons Attribution Non Commercial (CC BY-NC 4.0) license, which permits others to distribute, remix, adapt, build upon this work noncommercially, and license their derivative works on different terms, provided the original work is properly cited and the use is non-commercial. See: http:// creativecommons.org/licenses/by-nc/4.0/

\section{REFERENCES}

1. Cox AM, McKevitt C, Rudd AG, et al. Socioeconomic status and stroke. Lancet Neurol 2006;5:181-8.

2. Andersen G, Vestergaard K, Riis J, et al. Incidence of post-stroke depression during the first year in a large unselected stroke population determined using a valid standardized rating scale. Acta Psychiatr Scand 1994;90:190-5.

3. Mayer G, Jennum P, Riemann D, et al. Insomnia in central neurologic diseases--occurrence and management. Sleep Med Rev 2011;15:369-78.

4. Kripke DF, Klauber MR, Wingard DL, et al. Mortality hazard associated with prescription hypnotics. Biol Psychiatry 1998;43:687-93.

5. Rintoul AC, Dobbin MD, Nielsen S, et al. Recent increase in detection of alprazolam in Victorian heroin-related deaths. Med Aust 2013;198:206-9.

6. Merlo J, Ostergren PO, Mansson NO, et al. Mortality in elderly men with low psychosocial coping resources using anxiolytic-hypnotic drugs. Scand J Public Health 2000;28:294-7.

7. Kripke DF, Langer RD, Kline LE. Hypnotics' association with mortality or cancer: a matched cohort study. BMJ Open 2012;2:e000850.
8. Mallon L, Broman JE, Hetta J. Is usage of hypnotics associated with mortality? Sleep Med 2009;10:279-86.

9. Jennum $\mathrm{P}$, Baandrup L, Ibsen $\mathrm{R}$, et al. Increased all-cause mortality with use of psychotropic medication in dementia patients and controls: a population-based register study. Eur Neuropsychopharmacol 2015;25:1906-13.

10. Montastruc F, Gardette V, Cantet C, et al. Potentially inappropriate medication use among patients with Alzheimer disease in the REAL. FR cohort: be aware of atropinic and benzodiazepine drugs! Eur J Clin Pharmacol 2013;69:1589-97.

11. Lauterbach EC. Use of psychotropic medication in Alzheimer's disease is associated with more rapid cognitive and functional decline. Evid Based Med 2013;18:e50.

12. Rosenberg PB, Mielke MM, Han D, et al. The association of psychotropic medication use with the cognitive, functional, and neuropsychiatric trajectory of Alzheimer's disease. Int J Geriatr Psychiatry 2012;27:1248-57.

13. Vigen CL, Mack WJ, Keefe RS, et al. Cognitive effects of atypical antipsychotic medications in patients with Alzheimer's disease: outcomes from CATIE-AD. Am J Psychiatry 2011;168:831-9.

14. Hermann DM, Bassetti CL. Sleep-related breathing and sleep-wake disturbances in ischemic stroke. Neurology 2009;73:1313-22.

15. Jorge RE, Robinson RG, Arndt S, et al. Mortality and poststroke depression: a placebo-controlled trial of antidepressants. Am J Psychiatry 2003;160:1823-9.

16. Mead GE, Hsieh CF, Hackett M. Selective serotonin reuptake inhibitors for stroke recovery. JAMA 2013;310:1066-7.

17. Wildenschild C, Mehnert F, Thomsen RW, et al. Registration of acute stroke: validity in the Danish Stroke Registry and the Danish National Registry of Patients. Clin Epidemiol 2014;6:27-36.

18. Winkelmayer WC, Mehta J, Wang PS. Benzodiazepine use and mortality of incident dialysis patients in the United States. Kidney Int 2007;72:1388-93.

19. Allgulander C. Psychoactive drug use in a general population sample, Sweden: correlates with perceived health, psychiatric diagnoses, and mortality in an automated record-linkage study. Am J Public Health 1989;79:1006-10.

20. Baandrup $L$, Jennum $P$, Lublin $\mathrm{H}$, et al. Treatment options for residual insomnia in schizophrenia. Acta Psychiatr Scand 2013;127:81-2.

21. Bhalerao S, Seyfried LS, Kim HM, et al. Mortality risk with the use of atypical antipsychotics in later-life bipolar disorder. J Geriatr Psychiatry Neurol 2012;25:29-36.

22. Marras C, Gruneir A, Wang X, et al. Antipsychotics and mortality in Parkinsonism. Am J Geriatr Psychiatry 2012;20:149-58.

23. Kales $\mathrm{HC}$, Kim HM, Zivin K, et al. Risk of mortality among individual antipsychotics in patients with dementia. Am J Psychiatry 2012;169:71-9.

24. Honkola J, Hookana E, Malinen S, et al. Psychotropic medications and the risk of sudden cardiac death during an acute coronary event. Eur Heart J 2012;33:745-51.

25. Jackson CA, Mishra GD. Depression and risk of stroke in midaged women: a prospective longitudinal study. Stroke 2013;44:1555-60.

26. Majed B, Arveiler D, Bingham A, et al. Depressive symptoms, a time-dependent risk factor for coronary heart disease and stroke in middle-aged men: the PRIME Study. Stroke 2012;43:1761-7.

27. Nielsen TJ, Vestergaard M, Christensen B, et al. Mental health status and risk of new cardiovascular events or death in patients with myocardial infarction: a population-based cohort study. BMJ Open 2013;3:e003045.

28. Flaster M, Sharma A, Rao M. Poststroke depression: a review emphasizing the role of prophylactic treatment and synergy with treatment for motor recovery. Top Stroke Rehabil 2013;20:139-50.

29. Seifert CL, Poppert H, Sander D, et al. Depressive symptoms and the risk of ischemic stroke in the elderly--influence of age and sex. PLOS ONE 2012;7:e50803.

30. El Husseini N, Goldstein LB, Peterson ED, et al. Depression and antidepressant use after stroke and transient ischemic attack. Stroke 2012;43:1609-16.

31. Wang S, Linkletter C, Dore D, et al. Age, antipsychotics, and the risk of ischemic stroke in the Veterans Health Administration. Stroke 2012;43:28-31.

32. Pratt NL, Roughead EE, Ramsay E, et al. Risk of hospitalization for stroke associated with antipsychotic use in the elderly: a self-controlled case series. Drugs Aging 2010;27:885-93.

33. Glenn MB. Sudden cardiac death and stroke with the use of antipsychotic medications: implications for clinicians treating individuals with traumatic brain injury. J Head Trauma Rehabil 2010;25:68-70.

34. Sacchetti E, Trifirò G, Caputi A, et al. Risk of stroke with typical and atypical anti-psychotics: a retrospective cohort study including unexposed subjects. J Psychopharmacol 2008;22:39-46. 
35. Walker ER, McGee RE, Druss BG. Mortality in mental disorders and global disease burden implications: a systematic review and meta-analysis. JAMA Psychiatry 2015;72:334-41.

36. Tiihonen J, Mittendorfer-Rutz E, Torniainen M, et al. Mortality and cumulative exposure to antipsychotics, antidepressants, and benzodiazepines in patients with schizophrenia: an observational follow-up study. Am J Psychiatry 2015. [Epub ahead of print 7 Dec 2015].

37. Gueye PN, Lofaso F, Borron SW, et al. Mechanism of respiratory insufficiency in pure or mixed drug-induced coma involving benzodiazepines. J Toxicol Clin Toxicol 2002;40:35-47.

38. Wilton LV, Heeley EL, Pickering RM, et al. Comparative study of mortality rates and cardiac dysrhythmias in post-marketing surveillance studies of sertindole and two other atypical antipsychotic drugs, risperidone and olanzapine. J Psychopharmacol 2001;15:120-6.
39. Czekalla J, Kollack-Walker S, Beasley CM Jr. Cardiac safety parameters of olanzapine: comparison with other atypical and typical antipsychotics. J Clin Psychiatry 2001;62:35-40.

40. Schuld A, Kraus T, Haack M, et al. Obstructive sleep apnea syndrome induced by clonazepam in a narcoleptic patient with REM-sleep-behavior disorder. J Sleep Res 1999;8:321-2.

41. Sivertsen B, Omvik S, Pallesen S, et al. Sleep and sleep disorders in chronic users of zopiclone and drug-free insomniacs. J Clin Sleep Med 2009;5:349-54.

42. Henderson A, Wright M, Pond SM. Experience with 732 acute overdose patients admitted to an intensive care unit over six years. Med J Aust 1993;158:28-30.

43. Mortensen JK, Larsson $\mathrm{H}$, Johnsen SP, et al. Post stroke use of selective serotonin reuptake inhibitors and clinical outcome among patients with ischemic stroke: a nationwide propensity score-matched follow-up study. Stroke 2013;44:420-6. 